\title{
Omega-3/Omega-6 Fatty Acids for Attention Deficit Hyperactivity Disorder
}

\section{A Randomized Placebo-Controlled Trial in Children and Adolescents}

\author{
Mats Johnson \\ Sven Östlund \\ Gunnar Fransson \\ Björn Kadesjö \\ Christopher Gillberg \\ Göteborg University, Sweden
}

\begin{abstract}
Objective: The aim of the study was to assess omega $3 / 6$ fatty acids (eye q) in attention deficit hyperactivity disorder (ADHD). Method: The study included a randomized, 3-month, omega 3/6 placebo-controlled, one-way crossover trial with 75 children and adolescents (8-18 years), followed by 3 months with omega 3/6 for all. Investigator-rated ADHD Rating Scale-IV and Clinical Global Impression (CGI) scale were outcome measures. Results: A majority did not respond to omega $3 / 6$ treatment. However, a subgroup of $26 \%$ responded with more than $25 \%$ reduction of ADHD symptoms and a drop of CGI scores to the near-normal range. After 6 months, $47 \%$ of all showed such improvement. Responders tended to have ADHD inattentive subtype and comorbid neurodevelopmental disorders. Conclusion: A subgroup of children and adolescents with ADHD, characterized by inattention and associated neurodevelopmental disorders, treated with omega $3 / 6$ fatty acids for 6 months responded with meaningful reduction of ADHD symptoms. (J. of Att. Dis. 2008; XX(X) $x x-x x$ )
\end{abstract}

Keywords: attention deficit hyperactivity disorder; omega 3/6 fatty acids; developmental coordination disorder; reading writing disorder

$\mathrm{P}$ hospholipids containing polyunsaturated fatty acids, such as omega-3 and omega-6, are integral parts of the neuronal cell membranes of the brain, and it has been hypothesized that they may have a role in facilitating the transmission of signals between neurons (Sinn \& Wilson, 2006). During the past two decades, several studies have been conducted due to the growing interest in the role of nutrition in behavior and learning problems in children, especially regarding the omega-3 and omega-6 fatty acids. A link between attention deficit hyperactivity disorder (ADHD) symptoms, learning difficulties, and omega 3/6 fatty acid deficiency has been suggested (Stevens et al., 1995), and trials with supplementation of a combination of two omega-3 acids, eicosapentaenoic acid (EPA) and docosahexaenoic acid (DHA), in children with reading writing disorder (RWD), dyspraxia (i.e., motor coordination difficulties or developmental coordination disorder [DCD]), and ADHD-related symptoms have reported significant symptom reductions (Richardson \& Montgomery, 2005; Richardson \& Puri, 2002; Stevens et al., 2003). Trials with DHA only, however, have been less promising (Hirayama, Hamazaki, \& Terasawa, 2004;

Authors' Note: The authors acknowledge B. Areskoug, B. Börjesson, K. Hjalmarsson, M. Landgren, I. Matouskova, I. Olsson, and J. E. Månsson for their contributions, and the study was funded by Equazen UK Ltd. Address correspondence to Mats Johnson, MD, Department of Child and Adolescent Psychiatry, Institute of Neuroscience and Physiology, Göteborg University, Kungsgatan 12, 41118 Göteborg, Sweden; e-mail: mats.k.johnson@vgregion.se. 
Voight et al., 2001), suggesting that the presence of the EPA component might be important.

Two recently published placebo-controlled trials using the omega- 3 and omega- 6 fatty acid supplement Equazen eye q (hereafter termed omega 3/6) have been performed in Oxford-Durham, United Kingdom, and in Adelaide, South Australia. In the Oxford-Durham Study, 117 schoolchildren with DCD, aged 5 to 12 years, received omega $3 / 6$ or placebo, six capsules daily for 3 months, followed by 3 months of omega 3/6 at the same dose for all. Some degree of attention problems and hyperactivity was common in this group, but the children had no formal ADHD diagnosis. Significant reductions in ADHD symptoms were reported for the omega 3/6 group, measured on subscales of the Conners' Teacher Rating Scale-Long Version (Richardson \& Montgomery, 2005). The Australian study included 132 children, aged 7 to 12 years, who scored $+2 S D$ or more above population norms on the Conners' ADHD index, a subscale of the Conners' Parent Rating Scale-Long Version (Sinn \& Bryan, 2007). Thus, these children had ADHD index scores suggestive of ADHD, but the children were not formally clinically diagnosed for the study. They were randomized to receive 15 weeks' supplementation with omega 3/6 (either together with a micronutrient supplement containing vitamins C, B3, and B6, zinc, magnesium, etc., or omega 3/6 alone) or placebo, followed by 15 weeks with omega $3 / 6$ for all. Significant reductions in ADHD symptoms were found on the Conners' Parent Rating Scale but not on the corresponding teacher scale, and there was no significant difference with or without micronutrients.

The present study was launched with a view to replicating findings of the previous studies and to focus specifically on the treatment effects of omega 3/6 in young people diagnosed with ADHD. As defined in the Diagnostic and Statistical Manual of Mental Disorders (4th ed.; American Psychiatric Association [APA], 2000), ADHD has three subtypes (mainly inattentive, mainly hyperactive/impulsive, and combined), and to qualify for diagnosis, symptoms must cause significant functional impairment. The majority of children with ADHD also have some associated problems that can be diagnosed under the DSM-IV (Kadesjö \& Gillberg, 2001; Multimodal Treatment Study of Children With ADHD Cooperative Group, 1999). Difficulties with motor coordination (corresponding to DCD) and perception are common, and these symptom combinations are encompassed in the term DAMP (deficits in attention, motor control, and perception, which, in turn, correspond to ADHD with DCD). Many of these children have considerable problems with learning and reading or writing, and the most frequent other comorbidities are oppositional defiant disorder (ODD), varying degrees of symptoms within the autism spectrum, anxiety, depression, tics, and Tourette syndrome. Therefore, a special effort was made in the present study to make a detailed assessment of both the ADHD subtype and the occurring comorbidities so as to allow analysis of any difference in treatment outcome in diagnostic subgroups.

\section{Method}

\section{Objectives}

The primary objective was to assess whether supplementation with omega $3 / 6$ fatty acids was effective in reducing ADHD core symptoms in a clinical sample of children and adolescents with ADHD and to search for any differences in effect between well-defined subgroups in the sample based on detailed diagnosis of coexisting problems (comorbidity).

\section{Study Design}

This was a randomized placebo-controlled comprising two 3-month periods (Study Periods 1 and 2). The first 3month period was double-blind. The manufacturer of omega $3 / 6$ provided consecutively numbered identical bottles of which $50 \%$ contained active treatment and 50\% placebo in random order according to a code list that was not accessible to the investigators. The code was broken by a third party when all patients had completed the study after 6 months. At the start of Study Period 1, the patients were randomized to active treatment with omega $3 / 6$ in a dose of three capsules twice daily, corresponding to a daily dose of $558 \mathrm{mg}$ EPA, $174 \mathrm{mg}$ DHA (both are omega-3 fatty acids), $60 \mathrm{mg}$ gamma linoleic acid (an omega 6 fatty acid), and $10.8 \mathrm{mg}$ Vitamin $\mathrm{E}$, or to placebo (identical capsules containing olive oil). In Study Period 2, all patients were given (open) active treatment in the same dosage. This one-way crossover design (in preference to a two-way crossover) and length of treatment periods followed the model of the Oxford-Durham study (Richardson \& Montgomery, 2005) and was chosen due to the assumed slow turnover of the fatty acids in neuronal membranes (Bourre et al., 1993). The dosage is the same as in the Oxford-Durham and Adelaide studies because positive effects were reached without significant adverse events in these studies. Higher doses were considered unlikely to give more benefit based on the results of an earlier dose-response trial of treatment with EPA for depression in adults, in which the group which received 1 g/day showed a significantly better outcome than the groups treated with 2 or 4 g/day (A. Kelliher, personal communication, January 8, 2008; Peet \& Horrobin, 2002). 
Table 1

Demographics of Sample

\begin{tabular}{lccc}
\hline & \multicolumn{2}{c}{ Treatment Group } & Total $(N=75)$ \\
\cline { 2 - 4 } Patient Characteristics & Active $(n=37)$ & Placebo $(n=38)$ & $12(2.16)$ \\
\hline Age, years, $M(S D)$ & $11.8(2.14)$ & $12.2(2.19)$ & $48(64)$ \\
Age groups, $n(\%)$ & $27(56)$ & $21(44)$ & $27(36)$ \\
8-12 years & $10(37)$ & $17(63)$ & $64(85)$ \\
13-18 years & & & $11(15)$ \\
Gender, $n(\%)$ & $33(44)$ & $31(41)$ & \\
Male & $4(5)$ & $7(9)$ & $35(47)$ \\
Female & & & 0 \\
Attention deficit hyperactivity & $19(25)$ & $16(21)$ & $40(53)$ \\
$\quad$ disorder subtype, $n(\%)$ & 0 & 0 & $32(43)$ \\
Combined & $18(24)$ & $22(29)$ & $18(24)$ \\
Hyperactive/impulsive & & & $23(31)$ \\
Inattentive & $12(16)$ & $20(27)$ & $9(12)$ \\
Associated conditions, $n(\%)$ & $8(11)$ & $10(13)$ & $8(11)$ \\
Reading writing disorder & $10(13)$ & $13(17)$ & $11(15)$ \\
Oppositional defiant disorder & $3(4)$ & $6(8)$ & $2(3)$ \\
Developmental coordination disorder & $6(8)$ & $2(3)$ & $6(8)$ \\
Learning difficulties & $7(9)$ & $4(5)$ & $1(1)$ \\
Autistic traits & 0 & $2(3)$ & $4(5)$ \\
Autism-like condition or Asperger & $2(3)$ & 0 & \\
Tourette syndrome & $1(1)$ & & \\
Depression or anxiety & & & \\
Obsessive compulsive disorder & &
\end{tabular}

\section{Participants}

A total of 75 patients ( 64 boys and 11 girls), 35 with ADHD combined subtype and 40 with ADHD mainly inattentive subtype $(\mathrm{AD})$, were included in the present study (Table 1), which was conducted at three sites in southwest Sweden: the Child Neuropsychiatry Clinic in Göteborg, the General Child Psychiatry Clinic in Göteborg, and the Centre for Child Neurology in Mariestad. The study participants were recruited from among patients assessed and diagnosed at these clinics. Patients aged 8 to 18 years who met $D S M-I V$ criteria for a diagnosis of ADHD of any subtype, scoring at least $1.5 S D$ above the age norm for their diagnostic subtype using norms for the ADHD Rating Scale-IV-Parent Version (ADHDRS-IV; DuPaul, Power, Anastopoulos, \& Reid, 1998), were included in the study. The presence of any associated condition (comorbidity) was assessed by clinical interview according to DSM-IV criteria. Reading and writing ability was determined by standardized tests and RWD diagnosed according to $D S M-I V$ criteria for reading disorder and/or disorder of written expression (APA, 2000). The term learning difficulties (LD) used in this article corresponds to the $D S M-I V$ diagnosis of borderline intellectual functioning. Exclusion criteria for entry into the study were autism (however, autistic symptoms [AS] diagnosed in cases meeting three or more but not full symptom criteria for a diagnosis of autistic disorder, Asperger syndrome, or any of the other autism spectrum disorders was not an exclusion criterion), psychosis, bipolar disorder, mental retardation, uncontrolled seizure disorder, hyper- or hypothyroidism, significant other medical conditions, weight below $20 \mathrm{~kg}$, alcohol or drug abuse, or the use of any psychoactive drugs or omega 3 preparations in the past 3 months.

Of the 89 patients who were originally assessed with a view to enrollment in the trial, 7 could not be enrolled because they were shown to meet exclusion criteria: mild mental retardation $(n=4)$, autism $(n=1)$, sertraline treatment $(n=1)$, or meeting too few ADHD criteria $(n=1)$. Another 7 patients were excluded from the present study due to lack of ADHD-RS-IV and Clinical Global Impression (CGI) severity scale (Guy, 1976) data (see below) at the follow-up visits, leaving a total of 75 patients for analyses in this study. A large majority (78\%, 59/75) of the patients had at least one comorbid diagnosis (Table 1). Only 1 of the patients had previously been treated with medication for ADHD (methylphenidate). 


\section{Informed Consent and Ethics}

Written informed consent to participate was given by both the patient and the parent. The study was approved by the local ethical review board.

\section{Measures}

Clinical assessments were made at three visits at the sites: at baseline, 3 months (before switching to open treatment with omega 3/6), and 6 months.

Visit 1. At Visit 1, informed consent was signed, inclusion and exclusion criteria were assessed, and the investigators made a medical evaluation, including medical and psychiatric history, assessment of diagnosis and comorbidity through parent interview according to $D S M-I V$ criteria and of ADHD symptom severity by the ADHD-RS-IV and the CGI severity scale.

A number of other examinations and instruments were used, the results of some of which will be reported separately. These included a physical examination including height and weight, pulse and blood pressure, a neuromotor examination, using the medical motor examination (Kadesjö \& Gillberg, 1999), and an assessment of the general level of functioning with the Global Assessment of Functioning Scale. The questionnaires Swanson, Nolan, and Pelham (SNAP)-IV (Swanson, 1992), Five To Fifteen (FTF) Parent Scale (Kadesjö et al., 2004), and Brown's Attention Deficit Disorder Scale for Children and Adolescents (Brown, 1996) were completed by the parents; Brown's Self Report (Brown, 2001) was completed by the children; and the FTF Teacher Scale, Brown's Teacher Scale (Brown, 1996), and SNAP-IV were sent to the teachers for completion. Several neuropsychological tests were performed by a psychologist (S.Ö.) and reading-writing tests by a special education teacher (G.F.). At Visits 1-3, a blood sample was taken from all patients who agreed to this for analysis of the plasma fatty acid profile.

Visit 2. Visit 2 included the same examinations and instruments as Visit 1, with the exception of the FTF Parent and Teacher scales.

Visit 3. Visit 3 included all the same measures as Visit 1.

At Visits 2 and 3, the parents and children were also interviewed about current medical symptoms and any adverse events or side effects (open-ended questions).

Compliance was assessed by telephone parent reports, biweekly in Study Period 1 and monthly in Study Period 2. Compliance was defined as taking the prescribed dosage on more than $70 \%$ of the days in the interval.
Outcome measures. The primary outcome measures of ADHD symptoms were the investigator-rated ADHDRS-IV-Parent Version and the CGI severity scale. The ADHD-RS scale was originally validated as a parent- or teacher-rated scale, and U.S. norms for age and gender groups of children were developed (DuPaul et al., 1998). It was later validated as a clinician-rated parent interview to allow evaluation of symptom severity in multiple settings related to the clinicians' total experience of patients with ADHD, thus aiming for increased consistency (Faries, Yalcin, Harder, \& Heiligenstein, 2001), and has been used as a clinician-rated parent interview in several clinical trials with ADHD medications (Kratochvil et al., 2002; Michelson et al., 2001; Michelson et al., 2004). The raters of the ADHD-RS and CGI scales in the present study were pediatricians or child psychiatrists who had received training in using the scales.

The ADHD-RS-IV scores each of the 18 ADHD symptoms contained in the $D S M-I V$ diagnosis on a $0-3$ point scale, giving a maximum of 54 points. For clinically meaningful response, we used a definition that has been used in clinical trials with ADHD medications (e.g., Michelson et al., 2004), that is, a reduction of at least $25 \%$ of the symptom scores on the ADHD-RS scale.

The overall clinical impression of symptom severity and functional impairment was measured with the CGI severity scale, which is a clinician rating of the patient's symptom severity related to the clinician's total experience with ADHD patients, scored from 1 (normal, not ill) to 7 (among the most extremely ill patients).

\section{Statistical Analyses}

Treatment differences in mean changes of symptom scores from baseline to endpoint were assessed using the Wilcoxon test. All randomized patients with at least one postbaseline measurement were included in the analysis. Baseline was defined as the last measurement obtained at or before randomization, and endpoint was defined as the last measurement obtained after the randomization. Differences in responder rates were assessed using the chi-square test.

\section{Results}

\section{Attrition, Compliance, and Side Effects}

A total of 64 patients (78\% of all included, 54 boys and 10 girls) completed Study Period 1, and 59 (72\% of all, 49 boys and 10 girls) completed both study periods.

A total of 16 patients $(21 \%, 16 / 75)$ withdrew from the trial: 11 ( 3 active, 8 placebo) during Study Period 1 ( 7 were 
Table 2

Changes in ADHD-RS and CGI Scores for the Active or Placebo Groups After 3 Months and 6 Months

\begin{tabular}{|c|c|c|c|c|c|}
\hline Change in ADHD-RS and CGI Scores & Active $(M)$ & $S D$ & Placebo $(M)$ & $S D$ & Wilcoxon \\
\hline \multicolumn{6}{|l|}{ Study Period 1} \\
\hline ADHD-RS-IV total & -3.78 & 7.14 & -1.65 & 4.54 & 0.14 \\
\hline Inattention & -2.69 & 3.59 & -1.68 & 2.87 & 0.40 \\
\hline Hyperactivity/impulsivity & -1.28 & 5.10 & -0.13 & 2.38 & 0.25 \\
\hline CGI & -0.58 & 0.87 & -0.13 & 0.50 & 0.02 \\
\hline \multicolumn{6}{|l|}{ Study Period 2} \\
\hline ADHD-RS-IV total & -7.82 & 8.07 & -5.81 & 7.16 & 0.36 \\
\hline Inattention & -5.35 & 4.18 & -4.41 & 4.47 & 0.42 \\
\hline Hyperactivity/impulsivity & -3.32 & 5.30 & -1.52 & 3.60 & 0.08 \\
\hline CGI & -1.24 & 1.07 & -0.93 & 0.92 & 0.28 \\
\hline
\end{tabular}

Note: ADHD-RS = Attention Deficit Hyperactivity Disorder Rating Scale; CGI = Clinical Global Impression.

unmotivated to continue or had problems swallowing the capsules [ 1 active, 6 placebo], 3 had side effects in the form of dyspepsia, vomiting, or diarrhea [2 active, 1 placebo]), and the blinded code had to be broken for 1 patient (placebo) due to markedly increased irritability. Five $(5 \%, 5 / 75)$ withdrew during Study Period 2 (4 due to poor motivation and 1 due to diarrhea).

Compliance was generally high, with a mean of $93.4 \%$ (range $=74 \%-100 \%)$ for Study Period 1 and $93.3 \%$ for Study Period 2 (range $=75 \%-100 \%)$. All patients who completed a study period were also compliant for that period. Only those who withdrew from the study were noncompliant, and their results for the period in which they discontinued are not considered in the present analysis.

In addition to the 3 patients on active treatment who discontinued the study because of dyspepsia or diarrhea, 6 patients reported mild stomach discomfort or dyspepsia on active treatment. No other side effects were noted during the trial.

\section{Attention Deficit Hyperactivity Disorder Rating Scale-IV Scores}

At baseline, there were no significant differences between mean total ADHD-RS scores (active $=33.5, S D$ $=7.7$; placebo $=32.4, S D=8.0$ ), scores for inattention (active $=19.8, S D=3.2$; placebo $=19.5, S D=3.1$ ), or for hyperactivity/impulsivity (active $=14.2, S D=7.3$; placebo $=12.5, S D=6.9$ ). Overall, during Study Period 1 , there was a nonsignificant trend for greater reduction in ADHD-RS scores in the active group versus the placebo group, for total ADHD scores as well as for inattentive and hyperactive/impulsive subscores (Table 2). A similar trend was observed in Study Period 2 for the group which received active treatment in both study periods compared with those who received placebo in Study Period 1.

\section{Clinical Global Impression Results}

For the whole group, the mean CGI score was 4.67 (range $=4-6, S D=0.58$ ) at baseline, 4.06 (range $=2-6$, $S D=1.13)$ at Visit 2, and 3.38 (range $=1-6, S D=1.39)$ at Visit 3. The improvement in the active group at Visit 2 was significantly greater than in the placebo group (Table 2). For the Study Period 1 responders (see below) on active treatment, the CGI scores were reduced from moderate or marked severity $(M=4.6, S D=0.7, n=11)$ at baseline to the near-normal range, that is, $2-3$ ( $M=$ 2.6, $S D=0.52, n=8$ ), or from severe (6) to moderate (4) severity $(n=1)$. For the responders at the end of Study Period 2 compared with study start, the CGI scores had dropped to a similar range, that is, $1-3(M=2.4, S D=$ $0.7, n=28)$.

\section{Responders}

At the end of Study Period 1, 26\% (9/34, all boys) in the active group and $7 \%(2 / 30,1$ boy, 1 girl) in the placebo group were clinically meaningful responders, with more than $25 \%$ improvement in ADHD symptoms as measured on the ADHD-RS scale ( $p=.04$; Table 3 ). Four of the responders in the active group $(12 \%, 4 / 34)$ had more than $50 \%$ reduction of ADHD symptoms compared with none in the placebo group. At the end of Study Period 2, 47\% (28/59) were responders compared with study start, and among these were 7 patients (12\%) who had more than $50 \%$ symptom reduction. There appeared to be a difference between boys and girls, because all responders in the active group in Study Period 1 were boys, whereas the single responder among the girls was in the placebo group. However, the small sample of girls (10 girls completed Study Period 1) makes conclusions tentative, and at the end of Study 
Table 3

Patient Characteristics and Response Rates in Subgroups

\begin{tabular}{|c|c|c|c|c|c|}
\hline \multirow[b]{2}{*}{ Patient Characteristics } & \multirow[b]{2}{*}{$N$ (Baseline) } & \multicolumn{2}{|c|}{$\begin{array}{l}\text { Responders (\%) During } \\
0-3 \text { Months of Study } \\
\text { (No. of Responders } \\
\text { at Endpoint) }\end{array}$} & \multirow[b]{2}{*}{$p(\chi 2)$} & \multirow{2}{*}{$\begin{array}{c}\text { Responders }(\%) \\
\text { During 0-6 } \\
\text { Months of Study } \\
\text { (No. of Responders } \\
\text { at Endpoint }\end{array}$} \\
\hline & & Active & Placebo & & \\
\hline Total & 75 & $26(9 / 34)$ & $7(2 / 30)$ & .04 & $47(28 / 59)$ \\
\hline \multicolumn{6}{|l|}{ Gender } \\
\hline Male & 64 & $30(9 / 30)$ & $4(1 / 24)$ & .02 & $47(23 / 49)$ \\
\hline Female & 11 & $0(0 / 4)$ & $17(1 / 6)$ & $n s$ & $50(5 / 10)$ \\
\hline \multicolumn{6}{|l|}{ Age groups } \\
\hline $8-12$ years & 48 & $16(4 / 25)$ & $6(1 / 18)$ & $n s$ & $43(18 / 42)$ \\
\hline $13-18$ years & 27 & $56(5 / 9)$ & $9(1 / 11)$ & .02 & $59(10 / 17)$ \\
\hline \multicolumn{6}{|l|}{ Attention deficit hyperactivity disorder subtype } \\
\hline Combined & 35 & $17(3 / 17)$ & $8(1 / 13)$ & $n s$ & $36(10 / 28)$ \\
\hline Inattentive & 40 & $35(6 / 17)$ & $6(1 / 17)$ & .03 & $58(18 / 31)$ \\
\hline \multicolumn{6}{|l|}{ Associated conditions } \\
\hline Reading writing disorder & 34 & $43(6 / 14)$ & $12(2 / 17)$ & .05 & $52(15 / 29)$ \\
\hline DCD & 23 & $25(2 / 8)$ & $9(1 / 11)$ & $n s$ & $53(10 / 19)$ \\
\hline LD & 9 & $0(0 / 3)$ & $0(0 / 5)$ & $n s$ & $50(4 / 8)$ \\
\hline Autistic traits or ASP or ALC & 17 & $20(2 / 10)$ & $0(0 / 2)$ & $n s$ & $50(6 / 12)$ \\
\hline Any RWD, DCD, LD or autistic symptoms & 54 & $27(6 / 22)$ & $8(2 / 24)$ & $n s$ & $51(22 / 43)$ \\
\hline ODD & 18 & 0 & 0 & & 0 \\
\hline Conduct disorder & 2 & 0 & 0 & & 0 \\
\hline Depression or anxiety & 6 & 0 & 0 & & 0 \\
\hline
\end{tabular}

Note: $\mathrm{DCD}=$ developmental coordination disorder; $\mathrm{LD}=$ learning difficulties; $\mathrm{ASP}=$ asperger; $\mathrm{ALC}=$ autism-like condition; $\mathrm{RWD}=$ reading writing disorder; ODD = oppositional defiant disorder.

Period 2, the responder frequency was similar among boys and girls, $47 \%(23 / 49)$ versus $50 \%(5 / 10)$.

Analysis according to diagnostic subgroups revealed that responders were significantly more frequent in the AD group $(p=.03)$ than in the ADHD combined group (ns; Table 3). Responders tended to be more frequent among patients with a developmental disorder, that is, RWD $(p=.05)$, DCD, LD, or AS $(n s)$. In patients with other comorbidities (ODD, conduct disorder [CD], depression, anxiety, etc.) there were no responders.

\section{Discussion}

Overall, this is an essentially negative study. The double-blind randomized placebo-controlled trial demonstrated that for the whole group of children and adolescents with ADHD, omega 3/6 supplementation for 3 months was not statistically superior to placebo.

However, a closer look at variations in response within the group showed that there was a subgroup (26\% of all in the active group, $7 \%$ in the placebo group, $p=.04$ ) who responded with a clinically meaningful improvement in ADHD core symptoms, that is, a reduction of at least $25 \%$ on the ADHD Rating Scale-IV and a reduction of CGI scores to the near-normal range. Among these was a tendency for a small group (12\% in the active group, none in the placebo group, $n s$ ) who experienced a symptom reduction of more than $50 \%$. The results in the responder group were distinctly different from those of the nonresponders, who showed little or no change in symptom scores throughout the study. Another possible indication suggestive of a positive effect from active treatment was that discontinuation during the first 3 months tended to be slightly more common in the placebo group ( 3 active, 8 placebo, $n s$ ).

Comparison of treatment effect size in the present trial with that of the Oxford-Durham and Adelaide studies is limited by differences in the scales used as outcome measures. It is also possible that the patient samples were rather different. Our clinical patients were often severely affected, all had clinical AD or ADHD, and the majority had complex comorbidities.

A clinically meaningful response tended to be more frequent in the subgroup of children with associated neurodevelopmental comorbidity, that is, RWD, DCD, LD, or AS, and was lacking altogether in children with ODD, 
depression, or anxiety diagnoses. After 6 months (including at least 3 months of active treatment), $47 \%$ of all were responders. A small group (12\% of the boys, all in the Study Period 1 active group) experienced more than 50\% reduction in ADHD symptoms, making a substantial clinical difference in the daily functioning for these individuals. After 3 months, this was the case for 4 patients in the active group ( $3 \mathrm{AD}+\mathrm{RWD}, 1 \mathrm{AD})$. After 6 months, 7 patients (3 AD + RWD, 2 AD, 2 ADHD + DCD + RWD) had such marked improvement. This might tentatively be taken to mean that omega 3/6 treatment may be specifically effective for children with the phenotype of deficits in attention, motor control, and perception (DAMP), a finding that is in line with the results of the two previous studies in the field. The results indicate that it may be helpful to select patients with this phenotype and reading or writing difficulties for future research trials and treatment with omega $3 / 6$ fatty acids.

\section{Limitations}

Statistical power was limited by small sample size, particularly when it came to analysis of effects in clinical subgroups. It is possible that observed trends in treatment effects would reach significance with a larger sample.

Pill counts were not performed, which means that compliance might have been overestimated.

Most medication trials in ADHD have included mainly cases with the combined subtype. Our study is atypical in this respect given that about half of all participants had the mainly inattentive subtype of ADHD. Furthermore, there was a trend in the study that the children with this subtype might be more likely to respond to omega $3 / 6$. Thus, our findings cannot be generalized to apply to children with the combined subtype of ADHD.

The relatively infrequent clinic face-to-face follow-up visits in our protocol could be seen as a limitation affecting the recognition of early efficacy, but the slow turnover of omega 3/6 suggests that short-term effects should not be expected. Also, more frequent visits could have contributed toward increasing the risk of placebo effects.

\section{Conclusion}

This is the first double-blind randomized controlled trial of omega 3/6 for clinically diagnosed ADHD. The overall outcome was negative. More than half of all children with clinically impairing ADHD did not respond to 6 months of treatment with omega 3/6 fatty acids. However, about 1 in 8 patients in the study group showed considerable improvements (reduction of more than $50 \%$ of ADHD symptoms), and almost half the group responded to some degree after extension of the double-blind part of the study to include another 3 months of open treatment. Boys, and particularly those with ADHD inattentive subtype, DCD, RWD, and AS (often associated with the phenotype of the so-called DAMP syndrome), tended to be more likely to respond to the treatment. Side effects were few and rarely of clinical significance. However, a small subgroup reacted with dyspepsia severe enough to warrant termination of treatment.

\section{References}

American Psychiatric Association (2000). Diagnostic and statistical manual of mental disorders (4th ed.). Washington, DC: American Psychiatric Press.

Bourre, J. M., Bonneil, M., Clement, M., Dumont, O., Durand, G., Lafont, H., et al. (1993). Function of dietary polyunsaturated fatty acids in the nervous system. Prostaglandins Leukotrienes and Essential Fatty Acids, 48, 5-15.

Brown, T. E. (1996). Brown attention deficit disorder scales for adolescents and adults. San Antonio, TX: Psychological Corporation.

Brown, T. E. (2001). Brown attention deficit disorder scales for children and adolescents. San Antonio, TX: Psychological Corporation.

DuPaul, G. J., Power, T. J., Anastopoulos, A. D., \& Reid, R. (1998). ADHD rating scale-IV: Checklists, norms, and clinical interpretations. New York: Guilford.

Faries, D. E., Yalcin, I., Harder, D., \& Heiligenstein, J. H. (2001). Validation of the ADHD rating scale as a clinician administered and scored instrument. Journal of Attention Disorders, 5, $107-115$.

Guy, W. (1976). ECDEU assessment manual for psychopharmacology, revised. Bethesda, MD: U.S. Department of Health, Education, and Welfare.

Hirayama, S., Hamazaki, T., \& Terasawa, K. (2004). Effect of docosahexaenoic acid-containing food administration on symptoms of attention-deficit/hyperactivity disorder-a placebo-controlled double-blind study. European Journal of Clinical Nutrition, 58, 467-473.

Kadesjö, B., \& Gillberg, C. (1999). Developmental coordination disorder in Swedish 7-year-old children. Journal of the American Academy of Child and Adolescent Psychiatry, 38, 820-828.

Kadesjö, B., \& Gillberg, C. (2001). The comorbidity of ADHD in the general population of Swedish school-age children. Journal of Child Psychology and Psychiatry, 42, 487-492.

Kadesjö, B., Janols, L.-O., Korkman, M., Mickelsson, K., Strand, G., Trillingsgaard G., et al. (2004). The FTF (Five to Fifteen): the development of a parent questionnaire for the assessment of ADHD and comorbid conditions. European Child and Adolescent Psychiatry, 13(3), 3-13.

Kratochvil, C. J., Heiligenstein, J. H., Dittman, R., Spencer, T. J., Biederman, J., Wernicke, J., et al. (2002). Atomoxetine and methylphenidate treatment in children with ADHD: A prospective, randomized, open-label trial. Journal of the American Academy of Child and Adolescent Psychiatry, 41, 776-784. 
Michelson, D., Buitelaar, J. K., Danckaerts, M., Gillberg, C., Spencer, T. J., Zuddas, A., et al. (2004). Relapse prevention in pediatric patients with ADHD treated with Atomoxetine: A randomized, double-blind, placebo-controlled study. Journal of the American Academy of Child and Adolescent Psychiatry, 43, 896-904.

Michelson, D., Faries, D., Wernicke, J., Kelsey, D., Kendrick, K., Sallee, F. R., et al. (2001). Atomoxetine in the treatment of children and adolescents with attention-deficit/hyperactivity disorder: A randomized, placebo-controlled, dose-response study. Pediatrics 108, e83.

Multimodal Treatment Study of Children With ADHD Cooperative Group (1999). A 14-month randomized clinical trial of treatment strategies for attention-deficit/hyperactivity disorder. Archives of General Psychiatry, 56, 1073-1086.

Peet, M., \& Horrobin, D. F. (2002). A dose-ranging study of the effects of ethyl-eicosapentaenoate in patients with ongoing depression despite apparently adequate treatment with standard drugs. Archives of General Psychiatry, 59, 913-919.

Richardson, A. J., \& Montgomery, P. (2005). The Oxford Durham study: A randomized, controlled trial of dietary supplementation with fatty acids in children with developmental coordination disorder. Pediatrics, 115, 1360-1366.

Richardson, A. J., \& Puri, B. K. (2002). A randomized double-blind, placebo-controlled study of the effects of supplementation with highly unsaturated fatty acids on ADHD-related symptoms in children with specific learning difficulties. Progress in NeuroPsychopharmacology and Biological Psychiatry, 26, 233-239.

Sinn, N., \& Bryan, J. (2007). Effect of supplementation with polyunsaturated fatty acids and micronutrients on ADHD-related problems with attention and behaviour. Journal of Developmental and Behavioral Pediatrics, 28, 82-91.

Sinn, N., \& Wilson, C. (2006). Dietary supplementation with highly unsaturated fatty acids: Implications for interventions with persons with mental retardation from research on infant cognitive development, ADHD, and other developmental disabilities. International Review of Research in Mental Retardation, 32, 161-197.

Stevens, L. J., Zental, S. S., Deck, J. L., Abate, M. L., Watkins, B. A., Lipp, S. R., et al. (1995). Essential fatty acid metabolism in boys with attention-deficit hyperactivity disorder. American Journal of Clinical Nutrition, 62, 761-768.

Stevens, L. J., Zhang, W., Peck, L., Kuczek, T., Grevstad, N., Mahon, A., et al. (2003). EFA supplementation in children with inattention, hyperactivity, and other disruptive behaviours. Lipids, 38, 1007-1021.

Swanson, J. M. (1992). School-based assessments and interventions for ADD students. Irvine, CA: K. C. Publishing.

Voight, R. G., Llorente, A. M., Jensen, C. L., Fraley, K., Berretta, M. C., \& Heird, W. C. (2001). A randomized, double-blind, placebocontrolled trial of docosahexaenoic acid supplementation in children with attention-deficit/hyperactivity disorder. Journal of Pediatrics, 139, 189-196.

Mats Johnson, MD, is a clinician at the Child Neuropsychiatry Clinic in Göteborg. He is involved in research on interventions for ADHD, ODD, and neurodevelopmental disorders in children and adolescents.

Sven Östlund, BA, is a clinical psychologist at the Child Neuropsychiatry Clinic in Göteborg. He is involved in research on interventions for ADHD, ODD, and neurodevelopmental disorders in children and adolescents.

Gunnar Fransson, BA, is a special education teacher at the Child Neuropsychiatry Clinic in Göteborg. He is involved in research on interventions for ADHD, ODD, and neurodevelopmental disorders in children, adolescents, and young adults.

Björn Kadesjö, PhD, is the head of the Child Neuropsychiatry Clinic in Göteborg. He is involved in research on interventions and management of ADHD, ODD, and neurodevelopmental disorders in children, adolescents, and young adults.

Christopher Gillberg, $\mathrm{PhD}$, is a professor of child and adolescent psychiatry at Göteborg University. He is also with Strathclyde University, Glasgow, and the Institute of Child Health, London, UK. 